\title{
ANOTHER PROOF OF EXISTENCE OF MOMENTUM MAPPINGS
}

\author{
Dedicated to Professor Sihgeo Sasaki on his 70th birthday \\ By Kentaro Mikami
}

1. Momentum mappings for the symplectic action $G$ on a symplectic manifold are the group theoretical analogue of the linear and angular momentum associated with the translational and rotational invariance. Although momentum mappings are important in mechanics, not every symplectic action has a momentum mapping. We know some theorems concerning with the existence of (coadjoint equivariant) momentum mappings. These are as follows:

Theorem A ([4], [5]). If $H^{1}(g, R)=0$, then $G$ has a momentum mapping.

Theorem B (cf. [2], [4], [5]). If $H^{1}(\mathfrak{g}, R)=H^{2}(\mathrm{~g}, R)=0$, then $G$ has a coadjoint equivariant momentum mapping.

THEOREM C ([1]). If the symplectic form $\Omega=d \theta$ for some G-invariant 1-form $\theta$, then $G$ has a coadjoint equivariant momentum mapping.

THEOREM D (cf. [3]). If $G=G_{1} \underset{\sigma}{\rtimes} G_{2}$ where $G_{1}$ and $G_{2}$ have coadjoint equivariant momentum mappings, $G_{1}$ is connected and $H^{1}\left(g_{1}, R\right)=0$, then $G$ has a coadjoint equivariant momentum mapping.

Here $\mathfrak{g}$ and $g_{1}$ are the Lie algebras of $G$ and $G_{1}$ respectively.

According to [2], we consider a map $\Psi: M \rightarrow Z^{2}(\mathrm{~g})$ defined by $\Psi(m)=\phi_{m}^{*} \Omega$, where $\Omega$ is the symplectic form and $\phi_{m}$ is the orbit map through $m$, that is, $\psi_{m}(g)=g m$. In this paper, we will give another constructive and elementary proof to each of the above theorems by using the properties of $\Psi$.

Remark. In the original theorems of Theorem $\mathrm{B}$ and Theorem $\mathrm{D}$, the connectedness of the Lie group $G$ was needed because the coadjoint equivariancy of momentum mappings comes from the Lie algebra homomorphism. But our proofs are all right whether $G$ is connected or not.

2. Let $(M, \Omega)$ be a connected symplectic manifold, that is, $M$ is a connected smooth manifold with a non-degenerate closed 2 -form $\Omega$. Let $G$ be a symplectic

Received August 24, 1982 
action on $(M, \Omega)$, that is, $G$ is acting on $M$ and $\phi_{g}: M \rightarrow M: m \mapsto g m$ is a symplectomorphism, i.e., $\phi_{g}^{*} \Omega=\Omega$ for each $g$ in $G$. By $\phi_{m}$, we mean the orbit map $\psi_{m}: G \rightarrow M: g \mapsto g m$ through $m$. Let aut $(M, \Omega)$ be the Lie algebra of all Hamiltonian vector fields on $(M, \Omega)$, that is, $X \in \operatorname{aut}(M, \Omega)$ if and only if $L_{X} \Omega=0$. The symplectic action $G$ induces a Lie algebra homomorphism $\rho: \mathfrak{g} \rightarrow \operatorname{aut}(M, \Omega)$ defined by

$$
\rho(\xi)_{m}=\left(\phi_{m}\right)_{* e}(-\xi)=\frac{d}{d t}(\exp -t \xi m)_{t=0},
$$

where $\mathfrak{g}$ is the Lie algebra of $G$ consisting of left-invariant vector fields on $G$ and $e$ is the unit of $G$.

A momentum mapping for the symplectic action $G$ is a mapping $J: M \rightarrow \mathrm{g}^{*}$ (=the dual space of $\mathrm{g}$ ) such that

$$
d\langle J, \xi\rangle=\rho(\xi)\lrcorner \Omega .
$$

A momentum mapping $J: M \rightarrow \mathrm{g}^{*}$ for the symplectic action $G$ is coadjoint equivariant if and only if $J$ satisfies

$$
J(g m)=A d\left(g^{-1}\right)^{*} J(m)
$$

for each $g \in G$ and $m \in M$.

Since each left-invariant differential form on $G$ is completely determined by its value on the left-invariant vector fields, we can identify the space of leftinvariant $p$-forms on $G$ with the space of $p$-cochains of $g$. The exterior differentiation $d$ induces the coboundary operator $\delta$. According to [2], we consider a map $\Psi(m)=\phi_{m}^{*} \Omega$ on $M$. Then $\langle\Psi(m), \xi \wedge \eta\rangle=\Omega(\rho(\xi), \rho(\eta))$ holds. For each $g \in G$ and $m \in M$, we have

$$
\begin{aligned}
l_{g}^{* \Psi(m)} & =l_{g}^{*} \psi_{m}^{*} \Omega \\
& =\left(\phi_{m} l_{g}\right)^{*} \Omega \\
& =\left(\phi_{g} \psi_{m}\right) * \Omega \\
& =\phi_{m}^{*} \phi_{g}^{*} \Omega \\
& =\phi_{m}^{*} \Omega \\
& =\Psi(m), \\
\Psi(g m) & =\psi_{g}^{*} \Omega \\
& =\left(\phi_{m} r_{g}\right) * \Omega \\
& =r_{g}^{*} \psi_{m}^{*} \Omega \\
& =r_{g}^{*} \Psi(m) \\
& =A d\left(g^{-1}\right) * \Psi(m),
\end{aligned}
$$


where $l_{g}$ and $r_{g}$ are the left and right multiplications by $g$, and

$$
\delta \Psi(m)=d \psi_{m}^{*} \Omega=\phi_{m}^{*} d \Omega=0 .
$$

Differentiating the equation $\langle\Psi(m), \xi \wedge \eta\rangle=\Omega(o(\xi), \rho(\eta))_{m}$, we have

$$
v\lrcorner d\langle\Psi, \xi \wedge \eta\rangle=v\lrcorner-\rho[\xi, \eta]\lrcorner \Omega
$$

for any tangent vector $v$ on $M$. Thus we have

Proposition 1 ([2]). There is a coadjoint equivariant mapping $\Psi: M \rightarrow Z^{2}(\mathfrak{g})$ satisfying

(i) $\langle\Psi(m), \xi \wedge \eta\rangle=\Omega(\rho(\xi), \rho(\eta))_{m}$

and

(ii) $d\langle\Psi, \xi \wedge \eta\rangle=-\rho[\xi, \eta]\lrcorner \Omega$

for each $\xi$, $\eta$ in $\mathfrak{g}$, where $Z^{2}(\mathfrak{g})$ is the subspace of 2-cocycles of $\mathfrak{g}$.

Concerning to the 1-coboundary operator $\delta: C^{1}(\mathrm{~g}) \rightarrow C^{2}(\mathrm{~g})$, we have

Proposition 2. (i) $\delta$ is coadjont equivariant.

(ii) $\delta$ is injective if and only if $H^{1}(\mathrm{~g}, R)=0$.

(iii) $\delta\left(\mathrm{g}^{*}\right)=Z^{2}(\mathrm{~g})$ if and only if $H^{2}(\mathrm{~g}, R)=0$.

Proof. Choose any $\lambda \in C^{1}(\mathrm{~g})=\mathrm{g}^{*}$. For each $g \in G$ and $\xi$, $\eta \in \mathrm{g}$, we have

$$
\begin{aligned}
(A d(g) * \delta \lambda) \xi \wedge \eta & =\delta \lambda(A d(g) \xi \wedge A d(g) \eta)=-\lambda[A d(g) \xi, A d(g) \eta] \\
& =-\lambda(A d(g)[\xi, \eta])=-(A d(g) * \lambda)[\xi, \eta] \\
& =\left(\delta A d(g)^{*} \lambda\right) \xi \wedge \eta,
\end{aligned}
$$

thus (i) is proved. $\delta \lambda=0$ is equivalent to $\lambda[\mathrm{g}, \mathrm{g}]=0$. If $\delta \lambda=0$ and $H^{1}(\mathfrak{g}, R)=0$, that is, $g=[g, g]$, then $\lambda=0$. This shows that $\delta$ is injective. Conversely, assume that $\delta$ is injective. If $\mathfrak{g} \neq[\mathfrak{g}, \mathfrak{g}]$, then we can define 1 -cochain $\lambda$ such that $\delta \lambda=0$ and $\lambda \neq 0$. This contradicts to the injectivity of $\delta$. Thus (ii) is proved. (iii) is obvious because $H^{2}(\mathfrak{g}, R)=Z^{2}(\mathfrak{g}, R) / \delta\left(\mathrm{g}^{*}\right)$.

3. First we prove the following lemma, which suggests how to construct a momentum mapping from $\Psi$.

LEMMA. If $J$ is a momentum mapping for a symplectıc action $G$, then $\Psi-\delta J$ is constant on $M$.

Proof. From the definition of momentum mapping $J$ and (ii) of Proposition 1 , we have 


$$
\begin{aligned}
d\langle\delta J, \xi \wedge \eta\rangle & =d\langle J,-[\xi, \eta]\rangle \\
& =-\rho[\xi, \eta]\lrcorner \Omega \\
& =d\langle\Psi, \xi \wedge \eta\rangle
\end{aligned}
$$

for each $\xi, \eta$ in $g$. Since $M$ is connected, $\delta J-\Psi$ is constant on $M$.

(3.1) Proof of Theorem $B$. The assumption $H^{1}(\mathrm{~g}, R)=H^{2}(\mathrm{~g}, R)=0$, implies that $\delta: \mathrm{g}^{*} \rightarrow Z^{2}(\mathrm{~g})$ is bijective from (ii) and (iii) of Proposition 2. So we can define a mapping $J: M \rightarrow \mathrm{g}^{*}$ by

Since

$$
J(m)=\delta^{-1} \Psi(m)
$$

$$
\begin{aligned}
d\langle J,[\xi, \eta]\rangle & =-d\langle\delta J, \xi \wedge \eta\rangle \\
& =-d\langle\Psi, \xi \wedge \eta\rangle \\
& =\rho[\xi, \eta]\lrcorner \Omega
\end{aligned}
$$

and since $[\xi, \eta]$ 's generate $\mathfrak{g}, J$ is a momentum mapping. $J$ is also coadjoint equivariant because

$$
\begin{aligned}
J(g m) & =\delta^{-1} \Psi(g m)=\delta^{-1} A d\left(g^{-1}\right) * \Psi(m) \\
& =A d\left(g^{-1}\right)^{*} \delta^{-1} \Psi(m)=A d\left(g^{-1}\right)^{*} J(m) .
\end{aligned}
$$

(3.2) Proof of Theorem $C$. Since $\Omega=d \theta$, we have $\Psi(m)=\phi_{m}^{*} \Omega=\delta\left(\psi_{m}^{*} \theta\right)$, where $\phi_{m}^{*} \theta \in \mathrm{g}^{*}$. So we define $J(m)$ by $\phi_{m}^{*} \theta$. Coadjoint equivariancy of this mapping $J$ comes just the same way as the proof of that of $\Psi$ in Proposition 1 . For any vector field $X$ on $M$, we have

$$
\begin{aligned}
X\lrcorner d\langle J, \xi\rangle & \left.=L_{X}(\rho(-\xi)\lrcorner \theta\right) \\
& =-[X, \rho(\xi)]\lrcorner \theta-\rho(\xi)\lrcorner X\lrcorner d \theta-\rho(\xi)\lrcorner d(X\lrcorner \theta) \\
& =X\lrcorner \rho(\xi)\lrcorner \Omega .
\end{aligned}
$$

Thus $J(m)=\psi_{m}^{*} \theta$ is a coadjoint equivariant momentum mapping.

(3.3) Proof of Theorem A. (ii) of Proposition 1 implies that

$$
\langle\Psi, \xi \wedge \eta\rangle-\left\langle\Psi, \xi^{\prime} \wedge \eta^{\prime}\right\rangle=\text { constant on } M
$$

if $[\xi, \eta]=\left[\xi^{\prime}, \eta^{\prime}\right]$. Since $g=[g, g]$ by the assumption, we can define a map $J: M \rightarrow \mathrm{g}^{*}$ by

$$
\langle J(m),[\xi, \eta]\rangle=-\left\langle\Psi(m)-\Psi\left(m_{0}\right), \xi \wedge \eta\right\rangle
$$

where $m_{0}$ is any given point of $M$. Differentiating the above, we have

$$
\begin{aligned}
d\langle J,[\xi, \eta]\rangle & =-d\langle\Psi, \xi \wedge \eta\rangle \\
& =\rho[\xi, \eta]\lrcorner \Omega .
\end{aligned}
$$


Therefore $J$ is a momentum mapping.

(3.4) Proof of Theorem D. $G_{1}$ and $G_{2}$ have coadjoint equivariant momentum mappings, say $J_{1}$ and $J_{2}$. It is easily seen that $J=J_{1}+J_{2}$ is a momentum mapping for $G=G_{1} \rtimes G_{2}$. To prove the coadjoint equivariancy of $J$, it suffices only to show

$$
\left\langle J(g m)-A d\left(g^{-1}\right) * J(m), \eta\right\rangle=0
$$

for two cases (i) $g \in G_{2}$ and $\eta \in g_{1}$ or (ii) $g \in G_{1}$ and $\eta \in g_{2}$. For the case (i), since $H^{1}\left(g_{1}, R\right)=0$, we may assume $\eta=\left[\eta_{1}, \eta_{2}\right]$ where $\eta_{1}$ and $\eta_{2}$ are in $g_{1}$. Then we have

$$
\begin{aligned}
& \left\langle J(g m)-A d\left(g^{-1}\right)^{*} J(m),\left[\eta_{1}, \eta_{2}\right]\right\rangle \\
= & -\left\langle\Psi(g m)-A d\left(g^{-1}\right) * \Psi(m), \eta_{1} \wedge \eta_{2}\right\rangle \\
= & 0
\end{aligned}
$$

because of the coadjoint equivariancy of $\Psi$.

For the case (ii), consider the mapping

$$
G_{1} \ni g \mapsto\left\langle J(g m)-A d\left(g^{-1}\right)^{*} J(m), \eta\right\rangle,
$$

Differentiating this mapping to the direction $\xi \in \mathfrak{g}_{1}$, we have

$$
\begin{aligned}
& \rho(\xi)\lrcorner d\langle J, \eta\rangle-\langle J, \text { ad }(\xi) \eta\rangle \\
= & \rho(\xi)\lrcorner \rho(\eta)\lrcorner \Omega-\langle J,[\xi, \eta]\rangle \\
= & \Omega(\rho(\eta), \rho(\xi))+\langle\Psi, \xi \wedge \eta\rangle \\
= & 0 .
\end{aligned}
$$

Thus $\left\langle J(g m)-A d\left(g^{-1}\right)^{*} J(m), \eta\right\rangle$ is constant on $G_{1}$ if $G_{1}$ is connected and is 0 identically. This completes another proof of Theorem $\mathrm{D}$.

Acknowledgement. The author would like to express his sincere thanks to Professors Y. Hatakeyama, S. Tanno, H. Kitahara, T. Takahashi and S. Yorozu for their encouragement and helpful suggestions.

\section{REFERENCES}

[1] R. Abraham and J.E. Marsden, Foundations of mechanics, Benjamin, New York, 1978.

[2] V. Guillemin and S. Sternberg, Moments and reductions, Lecture Notes in Math., vol. 905, Springer-Verlag, Berlin and New York, 1980, pp. 52-65.

[3] K. Mikami, The existence of a coadjoint equivariant momentum mapping for a semidirect product, Proc. Amer. Math. Soc., vol. 82, 1981, pp. 465-469.

[4] N.R. Wallach, Symplectic geometry and Fourier analysis, Math. Sci. Press, 1970. 
[5] A. Weinstein, Lectures on symplectic manifolds, CBMS Regional Conf. Ser. in Math., no. 29, Amer. Math. Soc., 1977.

Department of Mathematics

Akita University

Akita 010, Japan 